\title{
华北盐渍化草地土壤净氮矿化速率对不同水平氮添 加的响应
}

徐小惠 1,2 刀华杰 ${ }^{1,3}$ 覃楚仪 ${ }^{4}$ 郝 杰 $^{3}$ 申 颜 ${ }^{1}$ 董宽虎 ${ }^{3}$ 王常慧 $1,3^{*}$

${ }^{1}$ 中国科学院植物研究所植被与环境变化国家重点实验室, 北京 $100093{ }^{2}{ }^{2}$ 中国科学院大学, 北京 $100049 ;{ }^{3}$ 山西农业大学草业学院, 山西太谷 $030801 ;$ 4武汉大学测绘学院, 武汉 430079

摘 要 对于养分贫瘦的盐渍化草地生态系统, 大气氮沉降如何影响土壤氮循环过程是一个目前尚未解决的问题。该研究在 位于华北地区山西省右玉县境内的盐渍化草地建立了一个模拟氮沉降的试验平台, 设置 8 个氮添加水平, 分别为 $0 、 1 、 2 、 4 、$ 8、16、24、32 g $\cdot \mathrm{m}^{-2} \cdot \mathrm{a}^{-1}(\mathrm{~N} 0 、 \mathrm{~N} 1 、 \mathrm{~N} 2 、 \mathrm{~N} 4 、 \mathrm{~N} 8 、 \mathrm{~N} 16 、 \mathrm{~N} 24 、 \mathrm{~N} 32)$, 生长季5-9月, 每月月初以喷施的方式等量添加 $\mathrm{NH}_{4} \mathrm{NO}_{3}$ 。 从2017年5月到2019年10月, 运用顶盖PVC管法每月一次进行净氮矿化速率的测定同时计算了净氮矿化速率对不同水平氮添 加的敏感性。主要结果表明: (1)高水平氮添加(N16、N24、N32)显著增加土壤无机氮库; (2)该盐渍化草地土壤氮矿化以硝化 作用为主, 经过3年氮添加以后, 高氮添加(N24、N32)显著促进了土壤净硝化速率, 并且不同氮添加水平在不同的月份和年份 中表现出差异性响应; (3)不同氮添加水平对土壤净氮矿化敏感性的影响在不同降水年份差异显著, 短期低水平氮添加提高了 土壤净氮矿化的敏感性, 而高水平氮添加降低土壤净氮矿化敏感性; (4)盐渍化草地土壤净氮矿化速率与土壤温度和水分呈正 相关关系, 与土壤 $\mathrm{pH}$ 呈负相关关系。因此, 在当前氮沉降增加的背景下, 北方盐渍化草地土壤氮矿化速率对低氮添加的敏感 性较高, 结合氮沉降的特点, 未来模型预测应该同时考虑氮沉降对盐渍化草地的可能影响。

关键词 氮添加; 盐渍化草地; 土壤净矿化速率; 敏感性; 硝化速率

徐小惠, 刀华杰, 覃楚仪, 郝杰, 申颜, 董宽虎, 王常慧 (2021). 华北盐渍化草地土壤净氮矿化速率对不同水平氮添加的响应. 植物生态学报, 45, 85-95. DOI: 10.17521/cjpe.2020.0153

\section{Response of soil net nitrogen mineralization to different levels of nitrogen addition in a saline-alkaline grassland of northern China}

XU Xiao-Hui ${ }^{1,2}$, DIAO Hua-Jie ${ }^{1,3}$, QIN Chu-Yi ${ }^{4}$, HAO Jie ${ }^{3}$, SHEN Yan ${ }^{1}$, DONG Kuan-Hu ${ }^{3}$, and WANG Chang-Hui ${ }^{1,3 *}$

${ }^{1}$ State Key Laboratory of Vegetation and Environmental Change, Institute of Botany, Chinese Academy of Sciences, Beijing 100093, China; ${ }^{2}$ University of Chinese Academy of Sciences, Beijing 100049, China; ${ }^{3}$ College of Grassland Science, Shanxi Agricultural University, Taigu, Shanxi 030801; and ${ }^{4}$ School of Geodesy and Geomatics, Wuhan University, Wuhan 430079, China

\section{Abstract}

Aims The salinized grassland ecosystem has low level of nutrients, and how increasing nitrogen (N) deposition affects $\mathrm{N}$ cycling has not been solved yet.

Methods An experimental platform was set up to simulate increasing $\mathrm{N}$ deposition in the saline alkaline grassland ecosystem in Youyu County, Shanxi Province, eight levels of $\mathrm{N}$ addition were designed, expressed by 0, 1, 2, 4, 8, 16, 24, $32 \mathrm{~g} \cdot \mathrm{m}^{-2} \cdot \mathrm{a}^{-1}$ (N0, N1, N2, N4, N8, N16, N24, N32). The same amount of ammonium nitrate $\left(\mathrm{NH}_{4} \mathrm{NO}_{3}\right)$ was sprayed at the beginning of each month during the growing seasons from May to September every year. From May of 2017, net N mineralization rate was measured once a month using top-cover PVC cylinders. The experiment has been conducted for three years from 2017 to 2019.

Important findings The results showed that, (1) High N addition levels (N16, N24, N32) significantly increased soil inorganic N pool. (2) Soil N mineralization in the salinized grassland ecosystem was dominated by nitrification. After three years of $\mathrm{N}$ addition, high levels of $\mathrm{N}$ addition (N24, N32) significantly promoted the rate of soil net nitrification, and different $\mathrm{N}$ levels showed different effects in different months and years. (3) The sensitivity of soil net $\mathrm{N}$ mineralization to different levels of $\mathrm{N}$ addition showed significant difference among three years with

收稿日期Received: 2020-05-15 接受日期Accepted: 2020-07-17

基金项目: 国家重点研发计划(2016YFC0500703和2017YFA0604802)和国家自然科学基金(31872406)。Supported by the National Key R\&D Program of China (2016YFC0500703 and 2017YFA0604802), and the National Natural Science Foundation of China (31872406).

* 通信作者Corresponding author (wangch@ibcas.ac.cn) 
different precipitation. Lower $\mathrm{N}$ addition improved the sensitivity of soil net $\mathrm{N}$ mineralization, while higher $\mathrm{N}$ addition reduced the sensitivity of soil net $\mathrm{N}$ mineralization. (4) There was a positive correlation between soil net $\mathrm{N}$ mineralization rate and soil temperature, water content, and a negative correlation between soil net $\mathrm{N}$ mineralization rate and soil $\mathrm{pH}$ value. The sensitivity of soil $\mathrm{N}$ cycle to $\mathrm{N}$ addition was higher at low level of $\mathrm{N}$ addition than at high level of $\mathrm{N}$ addition in the saline-alkaline grassland of north China. The influence of increasing $\mathrm{N}$ deposition on soil $\mathrm{N}$ cycle should be considered in process-based models in the future.

Key words nitrogen addition; saline-alkaline grassland; soil net nitrogen mineralization rate; sensitivity; soil nitrification rate

Xu XH, Diao HJ, Qin CY, Hao J, Shen Y, Dong KH, Wang CH (2021). Response of soil net nitrogen mineralization to different levels of nitrogen addition in a saline-alkaline grassland of northern China. Chinese Journal of Plant Ecology, 45, 85-95. DOI: 10.17521/cjpe.2020.0153

草地是面积最大的陆地生态系统, 我国拥有 393 万 $\mathrm{km}^{2}$ 天然草地, 占国土面积的 $41.7 \%$, 在全球 氮循环过程中发挥着重要的作用。我国盐渍化草地 面积930万 $\mathrm{hm}^{2}$, 华北地区是盐渍化草地密集区，也 是重要的草牧业生产基地和北方生态屏障, 为当地 居民提供着必要的生产和生活资料。该区养分贫㾉, 草地生产力低下, 是我国一个生态脆弱区, 由于自 然因素和人类活动的影响, 盐渍化草地面积呈现出 增加趋势, 严重制约了草地生态系统的可持续发展 (郭成源等, 2013)。

氮是植物生长必需的大量元素, 土壤中的氮通 常是以有机氮的形式存在, 有机氮必须经过氮矿化 过程转变为无机态氮才可被植物吸收利用(王常慧, 2005)。土壤氮矿化速率的高低决定土壤氮的有效性, 并影响生态系统生产力。陆地生态系统普遍受到氮 限制, 然而20世纪中叶以来, 随着工农业生产和人 民生活水平的提高, 大量活性氮(地球大气和生物 圈中所有生物、光化学和辐射活性氮化合物)及含氮 气体的排放增加(Galloway et al., 2008; Jia et al., 2014), 使得全球范围内的氮沉降不断增加 (Neff et al., 2002)。氮沉降增加会影响土壤氮矿化过程, 大量研究报道了氮增加对不同类型草地土壤氮矿 化的影响, 发现在沙质草地生态系统会显著增加净 氮矿化速率和硝化速率(于占源等, 2007); 而在内蒙 古羊草草原土壤累积矿化量在较低氮添加水平 $\left(5 \mathrm{~g} \cdot \mathrm{m}^{-1} \cdot \mathrm{a}^{-1}\right)$ 下高于较高氮添加水平 $\left(80 \mathrm{~g} \cdot \mathrm{m}^{-1} \cdot \mathrm{a}^{-1}\right)$ (张璐等, 2009), 在内蒙古草甸草原的研究中也发现, 在氮添加水平为 $20 \mathrm{~g} \cdot \mathrm{m}^{-1} \cdot \mathrm{a}^{-1}$ 时的土壤净氮矿化速率 大于 $50 \mathrm{~g} \cdot \mathrm{m}^{-1} \cdot \mathrm{a}^{-1}$ 添加水平(李阳等, 2019), 表明氮矿 化速率并不是随着氮添加水平增加而不断提高, 而 是存在对不同水平氮添加的响应阈值; 同时有研究 发现氮添加会降低(Aber \& Magill, 2004)土壤氮矿
化速率或者没有影响(Liu et al., 2015), 这些不同的 结果反映了不同生态系统土壤氮循环对氮输入响应 的差异。但是截至目前, 盐渍化草地土壤净氮矿化 速率对氮沉降响应的研究较少, 已有的研究表明外 源氮的输入通过影响土壤微生物的活性对土壤净氮 矿化速率(Zhang et al., 2013)产生影响; 盐渍化草地 高的土壤 $\mathrm{pH}$ 影响土壤微生物的群落结构与功能 (Chen et al., 2019)。因此对盐渍化草地土壤氮转化的 深入研究对于盐渍化草地的管理具有重要的意义。

我们于 2017年4月在山西省右玉县境内的盐渍 化草地设置了一个不同水平氮添加实验, 便于拓展 我们对养分贫痊条件下土壤氮矿化过程对外源氮输 入增加的认知。本研究拟回答两个科学问题: (1)盐 渍化草地土壤净氮矿化速率对短期不同水平氮添加 是否存在响应阈值? (2)盐渍化草地土壤净氮矿化速 率对短期不同水平氮添加响应的敏感性如何? 根据 以往研究我们做出以下假设: (1)盐渍化草地土壤净 氮矿化速率对不同的水平氮添加存在响应阈值, 阈 值范围在4-24 g $\cdot \mathrm{m}^{-2} \cdot \mathrm{a}^{-1}$ 。(2)盐渍化草地土壤净氮矿 化速率对短期不同水平氮添加响应的敏感性不同, 土壤净氮矿化速率对低氮添加的敏感性高于对高氮 添加的敏感性。

\section{1 材料和方法}

\section{1 实验设计与采样}

试验地点位于山西省右玉县 $\left(39.28^{\circ} \mathrm{N}, 112.29^{\circ}\right.$ E)山西右玉黄土高原草地生态系统定位观测研究站, 该地区海拔 $1348 \mathrm{~m}$, 年平均气温 $4.72{ }^{\circ} \mathrm{C}$, 年降水 量435 mm, 草地属于典型草地, 盐渍化程度较高, 土壤 $\mathrm{pH}$ 范围为9-10。植物群落以赖草 (Leymus secalinus)、碱茅(Puccinellia distans)、碱蒿(Artemisia anethifolia)等耐盐碱植物为优势种。土壤基本理化 
性质见表1。

试验期间2018年降水量与2017和2019年的降水 量有明显的差异, 2018年降水较2017、2019年明显增

表1 华北盐渍化草地样地土壤基本理化性质(平均值土标准误)

Table 1 Basic soil properties in the experiment sites in a saline-alkaline grassland sample plot of northern China (mean $\pm S E$ )

\begin{tabular}{lc}
\hline 土壤特性 Soil property & 2017年本底值 Original value of 2017 \\
\hline $\mathrm{pH}$ & $9.82 \pm 0.11$ \\
土壤全碳含量 $T C\left(\mathrm{~g} \cdot \mathrm{kg}^{-1}\right)$ & $7.01 \pm 1.02$ \\
土壤全氮含量 $T N\left(\mathrm{~g} \cdot \mathrm{kg}^{-1}\right)$ & $0.61 \pm 0.04$ \\
土壤全碳与全氮含量的比 $T C: T N$ & $11.57 \pm 1.93$ \\
\hline
\end{tabular}

$T C$, soil total carbon content; $T N$, soil total nitrogen content.
多(图1)。2017年的降水量为 $282.0 \mathrm{~mm}$, 集中在7、8 月 $(85.7$ 和 $39.2 \mathrm{~mm}) ; 2018$ 年的降水量为 $460.8 \mathrm{~mm}$, 同样也是集中在7、8月(202.8和137.4 mm); 2019年的 降水量为 $309.2 \mathrm{~mm}, 7$ 、8月降水量为 81.2 和 $55.4 \mathrm{~mm}$ 。

为了探究不同水平氮添加对盐渍化草地土壤净 氮矿化速率的影响, 设计氮添加实验, 实验于2017 年5月开始, 采用随机区组设计。根据研究所在地的 自然氮沉降水平 $2 \mathrm{~g} \cdot \mathrm{m}^{-2} \cdot \mathrm{a}^{-1}$ (Zhu et al., 2015), 设置8 个氮添加水平分别为 $0 、 1 、 2 、 4 、 8 、 16 、 24 、 32 \mathrm{~g} \cdot \mathrm{m}^{-2}$, 分别用N0、N1、N2、N4、N8、N16、N24、N32 表示, 实验设置如图2所示。每个处理6个重复, 共48

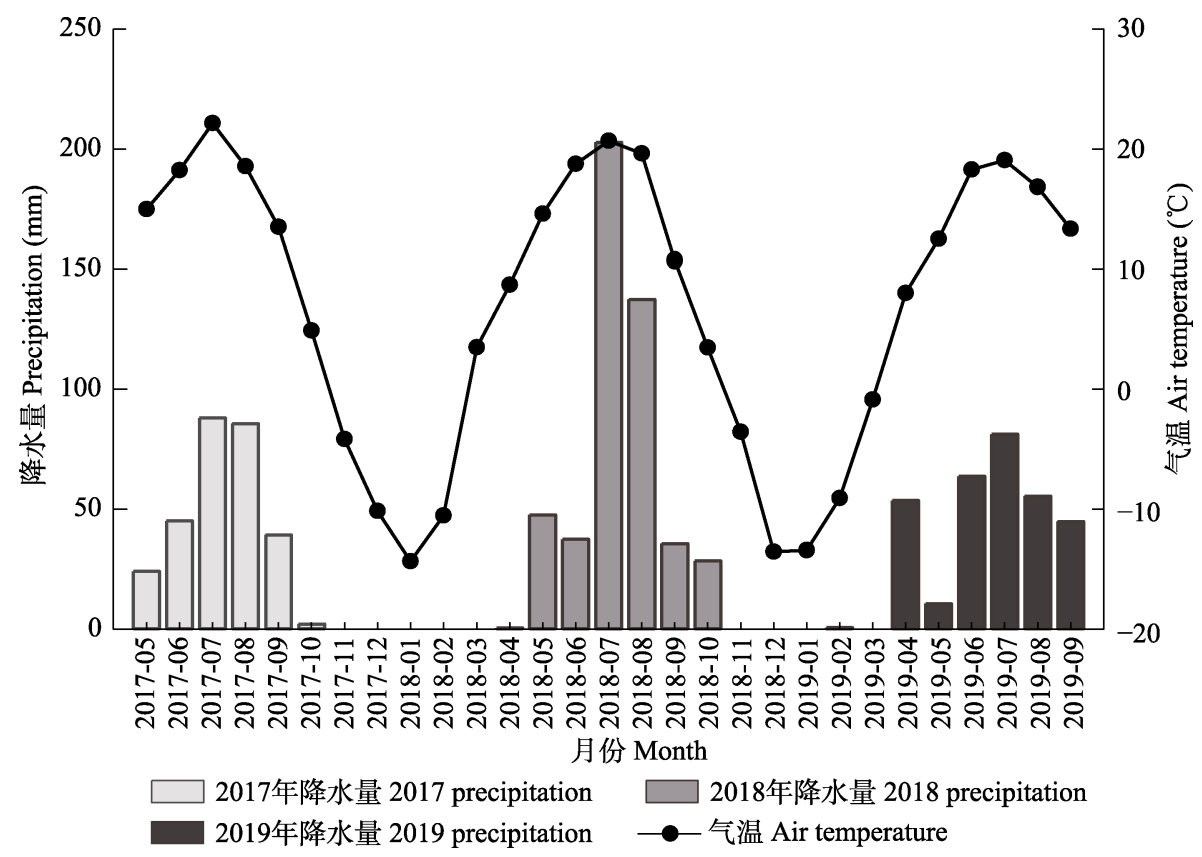

图1 华北盐渍化草地样地降水量和气温月动态变化。

Fig. 1 Monthly dynamics of precipitation and air temperature in a saline-alkaline grassland sample plot of northern China.

\begin{tabular}{|c|c|c|c|c|c|c|c|c|c|c|c|}
\hline No & $\mathrm{N} 32$ & N1 & N0 & N8 & N1 & N8 & $\mathrm{N} 24$ & $\mathrm{~N} 2$ & N1 & N16 & N8 \\
\hline N16 & $\mathrm{N} 24$ & $\mathrm{~N} 4$ & $\mathrm{~N} 2$ & N2 & N32 & N0 & N4 & N16 & N24 & $\mathrm{N} 24$ & N0 \\
\hline N2 & N8 & N16 & N32 & $\mathrm{N} 24$ & N0 & N1 & N32 & N8 & N4 & N1 & N32 \\
\hline N4 & N1 & N8 & N24 & N16 & N4 & N16 & N2 & N32 & N0 & N4 & N2 \\
\hline
\end{tabular}

图2 华北盐渍化草地试验地示意图。N0、N1、N2、N4、N8、N16、N24、N32分别表示氮添加水平分别为0、1、2、4、8、 16、24、32 $\mathrm{g} \cdot \mathrm{m}^{-2}$ 。

Fig. 2 Schematic diagram of experimental design in a saline-alkaline grassland of northern China. N0, N1, N2, N4, N8, N16, N24 and N32 indicated that the nitrogen addition levels were $0,1,2,4,8,16,24$ and $32 \mathrm{~g} \cdot \mathrm{m}^{-2}$, respectively. 
个小区，每个小区面积为 $6 \mathrm{~m} \times 9 \mathrm{~m}$, 小区之间设置 $2 \mathrm{~m}$ 缓冲带。野外实验于每年的5-9月的月初施加氮 肥 $\mathrm{NH}_{4} \mathrm{NO}_{3}$, 将不同剂量的 $\mathrm{NH}_{4} \mathrm{NO}_{3}$ 溶于 $20 \mathrm{~L}$ 的水中, 使用肩背式喷雾器均匀地喷酒到每个小区, 在对照 小区喷酒等量的水。采用顶盖PVC管法测定土壤净 氮矿化速率, 在每个处理样方砸下直径 $5 \mathrm{~cm}$ 、高度 $12 \mathrm{~cm}$ 的两个PVC矿化管, 每个月月中用直径 $3 \mathrm{~cm}$ 的土钻取两钻土混合装在自封袋中作为本月培养前 的土, 同时将两个矿化管的土取出混合装入自封袋 作为前一月培养后的土, 取出后将矿化管再次砸下 用于下一个月的矿化速率的测定, 具体方法见王常 慧(2005)。2017-2019年5-9月每个月月初施肥, 两周 后进行矿化速率监测。将取回的土壤分成两份, 一 份用于土壤含水量以及铵态氮、硝态氮的提取测定, 另一份荫蔽处自然风干, 用于测定土壤的各项理化 性质。

\section{2 土壤和植物指标的测定}

土壤温度利用数显电子温度计测定, 土壤含水 量用烘干称质量法测定; 利用 $\mathrm{pH}$ 电位计测定土壤 $\mathrm{pH}$ (土水体积比 $=1: 2.5$ ); 利用重铬酸钾氧化法测定 土壤的全碳含量; 利用凯氏定氮法测定土壤的全氮 含量(鲍士旦, 2000); 土壤的铵态氮、硝态氮用 0.5 $\mathrm{mol} \cdot \mathrm{L}^{-1}$ 硫酸钾溶液以水土体积比 $5: 1$ 浸提后, 浸提 液用全自动流动分析仪(FIAstar 5000, Foss Tecator, Denmark)测定; 利用收获法测定地上生物量, 8月份 在每个小区内固定样方中, 用剪刀将物种剪下分类 装入信封并收集凋落物, 在 $65{ }^{\circ} \mathrm{C}$ 烘干至恒质量, 称 量。地下净初级生产力采用根系内生长法测定, 4月 初在小区内钻取0-10、10-20、20-40 cm的土壤, 过 $2 \mathrm{~mm}$ 篮去除原有的根后, 按原土层回填, 10月末取 出, 用清水冲洗, 晾干, 烘干至恒质量, 计算根当年 的生长量, 具体方法见孙建平(2018)。

\section{3 计算方法}

净氮矿化速率的计算用培养后的无机氮 $\left(\mathrm{NH}_{4}^{+}-\right.$ $\left.\mathrm{N}+\mathrm{NO}_{3}^{-}-\mathrm{N}\right)$ 含量减培养前无机氮 $\left(\mathrm{NH}_{4}^{+}-\mathrm{N}+\mathrm{NO}_{3}^{-}-\mathrm{N}\right)$ 含量除以培养时间, 净硝化速率的计算用培养后的 硝态氮 $\left(\mathrm{NO}_{3}^{-}-\mathrm{N}\right)$ 含量减培养前 $\mathrm{NO}_{3}^{-}-\mathrm{N}$ 含量除以培养 时间, 公式如下:

$$
\begin{aligned}
& N R_{\text {min }}=\frac{\Delta C\left(\mathrm{NO}_{3}^{-}-\mathrm{N}\right)+\Delta C\left(\mathrm{NH}_{4}^{+}-\mathrm{N}\right)}{\Delta T} \\
& N R_{\text {nit }}=\frac{\Delta C\left(\mathrm{NO}_{3}^{-}-\mathrm{N}\right)}{\Delta T}
\end{aligned}
$$

$$
\begin{aligned}
& \Delta T=T_{i+1}-T_{i} \\
& \Delta C\left(\mathrm{NO}_{3}^{-}-\mathrm{N}\right)=C\left(\mathrm{NO}_{3}^{-}-\mathrm{N}\right)_{i+1}-C\left(\mathrm{NO}_{3}^{-}-\mathrm{N}\right)_{i} \\
& \Delta C\left(\mathrm{NO}_{3}^{-}-\mathrm{N}\right)=C\left(\mathrm{NH}_{4}^{+}-\mathrm{N}\right)_{i+1}-C\left(\mathrm{NH}_{4}^{+}-\mathrm{N}\right)_{i}
\end{aligned}
$$

式中, $N R_{\min }$ 表示净氮矿化速率, $N R_{\mathrm{nit}}$ 表示净硝化 速率, $\Delta C\left(\mathrm{NO}_{3}^{-}-\mathrm{N}\right)$ 表示净硝态氮增加量, $\Delta C\left(\mathrm{NH}_{4}^{+}-\mathrm{N}\right)$ 表示净铵态氮增加量, $C\left(\mathrm{NO}_{3}^{-}-\mathrm{N}\right)_{i+1}$ 表示培养后的硝态氮含量, $C\left(\mathrm{NO}_{3}^{-}-\mathrm{N}\right)_{i}$ 表示培养前 的硝态氮含量, $C\left(\mathrm{NH}_{4}^{+}-\mathrm{N}\right)_{i+1}$ 表示培养后的铵态氮 含量, $C\left(\mathrm{NH}_{4}^{+}-\mathrm{N}\right)_{i}$ 表示培养前的铵态氮含量, $\Delta T$ 为培养时间间隔。

根据年平均初级生产力对降水敏感性的计算方 法(Hsu et al., 2012), 本研究利用了一个公式来表示 不同施氮水平下, 土壤的净氮矿化对氮添加的敏感 性 $\left(N_{\mathrm{sen}}\right)$, 该公式计算如下:

$$
N_{\text {sen }}=\frac{\left(X_{i}-X_{\mathrm{ck}}\right) / X_{\mathrm{ck}}}{N_{\text {lev }} / N_{\text {dep }}}
$$

式中, $X_{i}$ 处理后测定的净氮矿化速率, $X_{\mathrm{ck}}$ 为对照 测定的净氮矿化速率, $N_{\text {lev }}$ 为施氮水平, $N_{\text {dep }}$ 为该 研究地点氮沉降值。若 $N_{\text {sen }}>0$, 说明此水平氮添加 对土壤净氮矿化为促进作用; 若 $N_{\text {sen }}<0$, 则说明 此水平氮添加对土壤净氮矿化为抑制作用, 但 $N_{\mathrm{sen}}$ 的大小与正负无关。

\section{4 数据的处理和分析}

采用SPSS 21.0对数据进行单因素方差分析和 最小显著性差异 $(L S D)$ 法检验, 分析不同氮添加水 平对铵态氮、硝态氮、净氮矿化速率以及净氮矿化 敏感性的影响, 采用相关性分析分析净氮矿化速率 与土壤 $\mathrm{pH}$ 、土壤温度、水分以及地下生物量的相关 性, 利用双因素方差分析和 $L S D$ 检验, 分析年份和 氮添加水平对净铵化、净硝化以及净氮矿化速率影 响的差异, 利用SigmaPlot 12.5作图。

\section{2 结果}

\section{1 不同水平氮添加对土壤铵态氮、硝态氮和无机 氮含量的影响}

土壤铵态氮、硝态氮和总的无机氮含量随着氮 添加量的增加显著上升 $(p<0.05$, 图3), 但是并没有 随着施肥年限的增加出现累积效应。与对照相比, 氮添加后2017年的铵态氮含量变化范围为 $0.38-$ $0.62 \mathrm{mg} \cdot \mathrm{kg}^{-1}$, N2、N8、N16处理使得铵态氮含量显 著增加 $(p<0.05)$, 分别增加61.70\%、51.09\%、30.87\%;

www.plant-ecology.com 

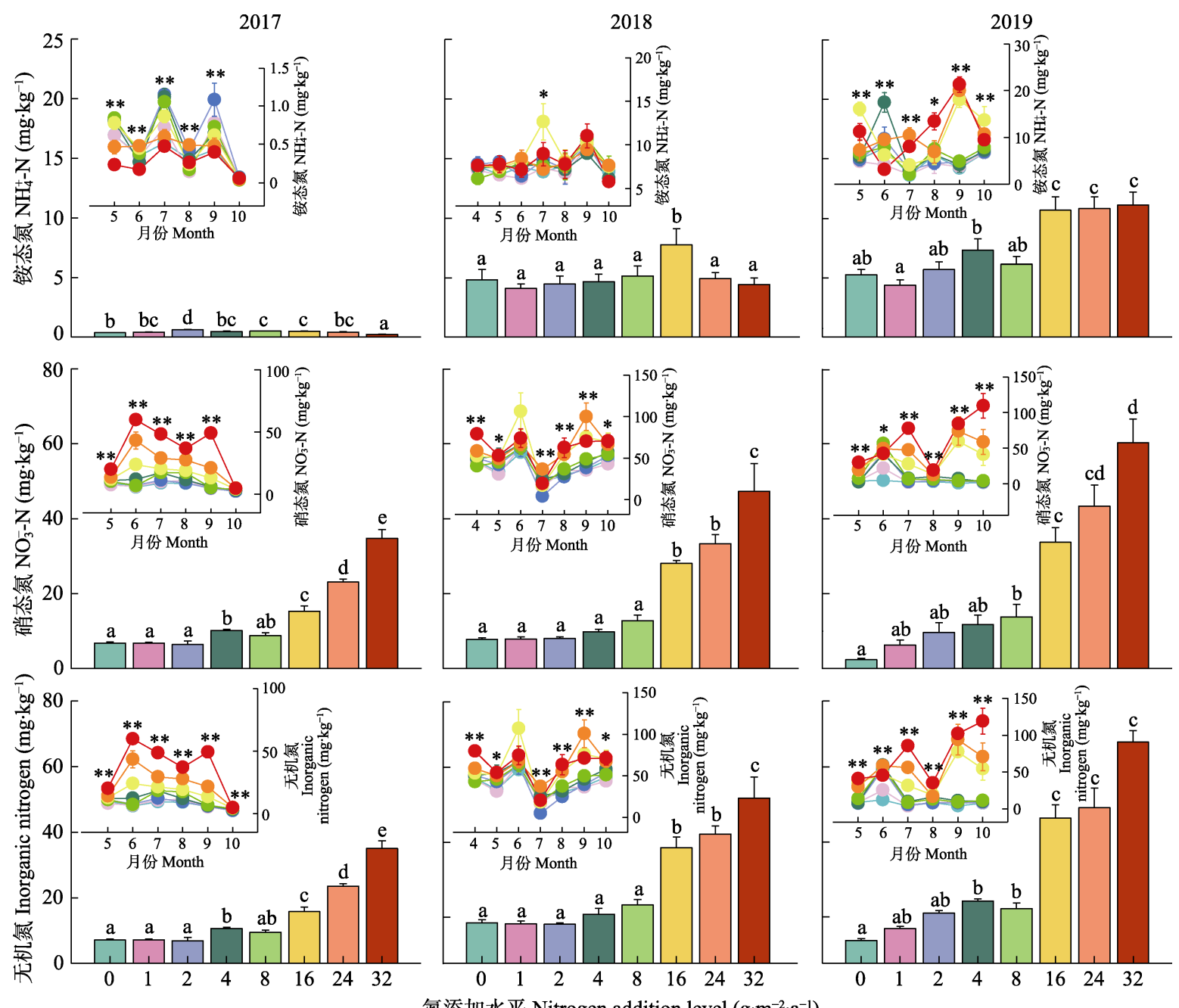

图3２017-2019年华北盐渍化草地不同氮添加水平铵态氮、硝态氮和无机态氮含量变化(平均值+标准误)。不同小写字母 表示差异显著 $(p<0.05)$ 。*表示施肥和对照的差异性在 0.05 水平上显著; **表示施肥和对照差异性在 0.01 水平上显著。小图 中不同颜色对应柱状图的不同氮添加水平。

Fig. 3 Changes of soil $\mathrm{NH}_{4}^{+}-\mathrm{N}, \mathrm{NO}_{3}^{-}-\mathrm{N}$ and inorganic nitrogen concentration under different levels of nitrogen addition in 2017-2019 of a saline-alkaline grassland of northern China (mean + SE). Different lowercase letters indicate significant differences $(p<0.05)$. * means the difference is significant at the level of $p<0.05$, and $* *$ means the difference is significant at the level of $p<0.01$. Different colors in the small picture correspond to different nitrogen addition levels in the histogram.

2018年氮添加水平为N16时使铵态氮含量显著增加 $(p<0.05)$, 增加了61.31\%; 2019年从N16开始铵态 氮含量显著增加 $(p<0.05)$, 分别增加了 $103.25 \%$ 、 $106.07 \%$ 和 $111.64 \% 。 2017-2019$ 年, 硝态氮和无机氮 含量随着施氮水平的增加而增加, 氮添加水平为 N16处理时会显著增加土壤的无机氮含量 $(p<0.05)$, 并且随着施氮水平的增加而显著增加, 2019年的铵 态氮、硝态氮和无机氮含量高于2017和2018年的含 量。硝态氮含量显著高于铵态氮含量 $(p<0.05)$, 可 以看出无机氮含量的变化主要是由硝态氮含量的变 化引起。

2017年，铵态氮含量呈现出波浪形的趋势，5-9 月施肥会极显著提高铵态氮的含量 $(p<0.01), 7$ 月铵
态氮含量最高, 不同氮处理的铵态氮含量的变化范 围是0.47-1.14 mg $\mathrm{kg}^{-1} ; 2018$ 年4-7月铵态氮含量降 低, 但氮添加后会显著提高铵态氮的含量 $(p<0.05)$, 7-8月含量升高, 其中4月N16处理铵态氮含量最高, 为 $0.76 \mathrm{mg} \cdot \mathrm{kg}^{-1} ; 2019$ 年8-10月, N0-N8处理的季节 动态变化呈现相似的趋势, N16、N24、N32处理的 氮添加处理后季节变化一致, 前者含量先降低后升 高, 后者铵态氮含量先增加后降低, 9月N32处理后 铵态氮含量最高, 为 $21.30 \mathrm{mg} \cdot \mathrm{g}^{-1}$ 。2017和2018年硝 态氮含量在7月的N32处理氮添加水平下最高, 生长 季不同月份之间全部为N16、N24、N32处理氮添加 的硝态氮含量显著高于对照处理 $(p<0.01)$ 。2019年 硝态氮含量7-8月显著降低 $(p<0.05), 8-9$ 月显著增 
加 $(p<0.05), 9$ 月的N32处理下硝态氮含量最高。 2017-2019年, 不同水平氮添加后无机氮含量的季 节动态变化与硝态氮含量变化一致。

\section{2 不同氮添加水平处理下土壤净氮矿化速率的生 长季季节动态}

通过双因素方差分析发现年际效应和不同氮添 加水平会显著影响铵化速率 $(p<0.05, p<0.001$; 表 $2)$ 、硝化速率 $(p<0.001)$ 以及净氮矿化速率 $(p<0.05$, $p<0.001)$, 同时年份和氮添加处理的交互作用对铵 化速率 $(p<0.05)$ 、硝化速率 $(p<0.001)$ 以及净氮矿化 速率 $(p<0.05)$ 也具有显著影响。

2017-2019年3年的净氮矿化速率的月动态变化 不一致(图4), 但是2019年的净矿化速率要显著高于 2017、2018年 $(p<0.05), 2018$ 年5月, 与对照相比, 氮 添加(N24、N32)后显著增加了土壤的净氮矿化速率 $(p<0.01), 8$ 月 $24 、 \mathrm{~N} 32$ 处理显著低于对照 $(p<$ $0.01)$ 。

2019年7、9和10月氮添加后显著改变了土壤的 净氮矿化速率 $(p<0.05), \mathrm{N} 16 、 \mathrm{~N} 24 、 \mathrm{~N} 32$ 处理下的 净氮矿化速率在7和9月显著高于对照处理的净氮矿 化速率 $(p<0.01)$, 而10月N16、N24、N32处理下的 净氮矿化速率显著低于对照处理 $(p<0.01)$ 。
不同水平氮添加显著影响土壤净硝化速率和净 矿化速率 $(p<0.05)$ (图5), 净硝化速率和净矿化速率 在2017-2019年对不同水平氮添加的响应不同, 2017 年不同水平氮添加与对照相比净硝化速率和净氮矿 化速率都无显著差异 $(p>0.05)$, 氮添加对土壤的净 硝化速率和净氮矿化速率在短期内没有影响, 2018 年N16处理显著降低了土壤净矿化速率 $(p<0.05)$, 与对照相比, 降低了 $112.15 \%$, 净硝化速率在不同 水平氮添加后与对照之间没有显著差异 $(p>0.05)$; 2019年N24、N32处理显著增加土壤的净硝化速率 $(p<0.05)$, 与对照相比, 分别增加了 $232.07 \%$ 和 206.03\%。

\section{3 不同氮添加水平对土壤净氮矿化速率敏感性的 影响}

净氮矿化速率对不同水平氮添加的敏感性不同 (图6), 低水平氮添加的敏感性高于高水平氮添加, 2019年的净氮矿化速率较2017和2018年对低水平氮 添加的敏感性更高, 2019年净氮矿化速率的敏感性 在N1处理时显著高于其他处理 $(p<0.05) ; 2018$ 年N2 处理的敏感性显著高于N8处理的敏感性 $(p<0.05)$; 2017年各氮添加处理对于净氮矿化速率的敏感性没 有显著差异 $(p>0.05)$ 。此外, 净氮矿化速率对不同

表2 不同氮添加水平和处理时间对华北盐渍化草地土壤铵化速率、硝化速率、净氮矿化速率影响的双因素方差分析

Table 2 Two-way ANOVA on the effects of nitrogen addition and year on soil net ammonification, nitrification and net nitrogen mineralization rates of the salinized grassland of northern China

\begin{tabular}{|c|c|c|c|c|c|c|}
\hline \multirow[t]{2}{*}{$\begin{array}{l}\text { 因素 } \\
\text { Factor }\end{array}$} & \multicolumn{2}{|c|}{$\begin{array}{c}\text { 铵化速率 } \\
\text { Ammonification rate }\end{array}$} & \multicolumn{2}{|c|}{$\begin{array}{c}\text { 硝化速率 } \\
\text { Nitrification rate }\end{array}$} & \multicolumn{2}{|c|}{$\begin{array}{c}\text { 净氮矿化速率 } \\
\text { Net nitrogen mineralization rate }\end{array}$} \\
\hline & F & $p$ & F & $p$ & F & $p$ \\
\hline 年份 Year (Y) & 6.167 & $<0.05$ & 35.338 & $<0.001$ & 5.275 & $<0.05$ \\
\hline 氮添加 Nitrogen addition (N) & 7.879 & $<0.001$ & 12.592 & $<0.001$ & 9.455 & $<0.001$ \\
\hline 年份×氮添加 $(\mathrm{Y} \times \mathrm{N})$ & 3.664 & $<0.05$ & 14.516 & $<0.001$ & 2.315 & $<0.05$ \\
\hline
\end{tabular}

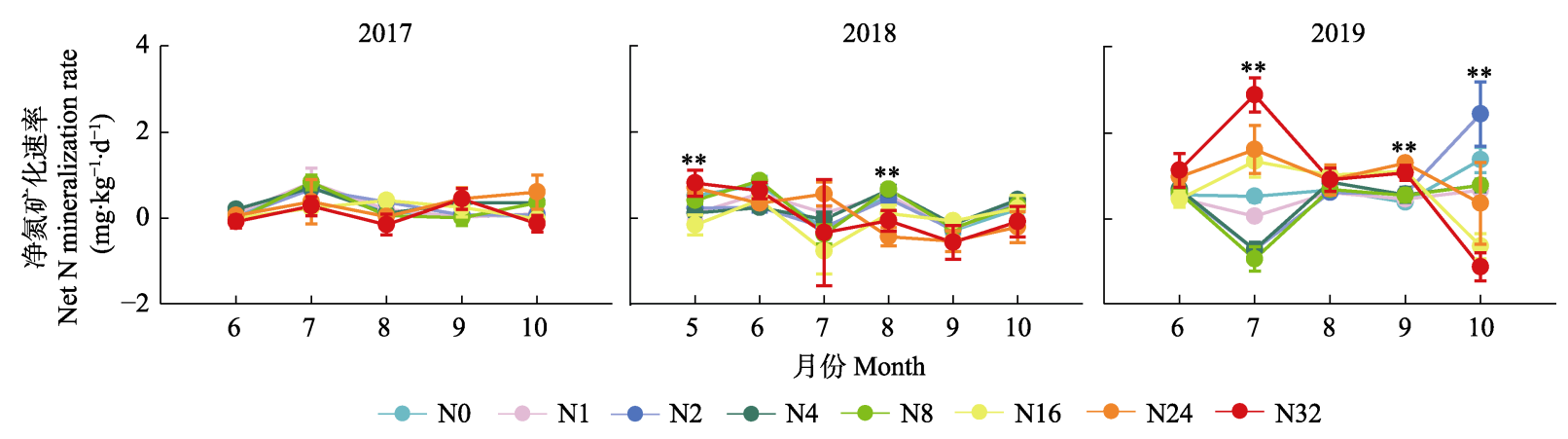

图4 华北盐渍化草地不同氮添加水平土壤净氮矿化速率的季节动态(平均值土标准误)。**表示差异性在 0.01 水平上显著。 N0、N1、N2、N4、N8、N16、N24、N32分别表示氮添加水平分别为0、1、2、4、8、16、24、32 $\mathrm{g} \cdot \mathrm{m}^{-2}$ 。

Fig. 4 Seasonal dynamics of soil net $\mathrm{N}$ mineralization rates under different levels of nitrogen addition in a saline-alkaline grassland of northern China (mean $\pm S E$ ). ** means the difference is significant at the level of $0.01 . \mathrm{N} 0, \mathrm{~N} 1, \mathrm{~N} 2, \mathrm{~N} 4, \mathrm{~N} 8, \mathrm{~N} 16, \mathrm{~N} 24$ and N32 indicated that the nitrogen addition levels were $0,1,2,4,8,16,24$ and $32 \mathrm{~g} \cdot \mathrm{m}^{-2}$, respectively. 
水平氮添加的敏感性在不同年际之间差异显著, 2018年净氮矿化速率对氮添加的敏感性小于2017和 2019年。

\section{4 影响土壤净氮矿化速率的生物和非生物因子}

不同水平氮添加对净氮矿化的影响因素不同 (图7), N0-N24的施肥处理中净氮矿化速率与土壤 温度有关, 并呈现显著正相关关系 $(p<0.05)$, N8和 $\mathrm{N} 16$ 施肥处理与土壤湿度呈显著正相关关系 $(p<$ $0.05)$, 在 $\mathrm{N} 1$ 处理时与土壤 $\mathrm{pH}$ 呈显著负相关关系 $(p<$ $0.05)$, 在 $\mathrm{N} 32$ 处理时与地下生物量呈显著正相关关 系 $(p<0.05)$ 。

\section{3 讨论}

\section{1 土壤净氮矿化速率对不同水平氮添加的响应} 阈值

土壤中的无机氮以铵态氮和硝态氮形式存在, 是植物生长利用的主要养分。草地土壤无机氮含量 的高低是由无机氮的输入(如氮添加)、氮的转化及 植物吸收和利用共同决定的(Zhang et al., 2012; Liu et al., 2015)。我们发现, 随着施氮水平的升高, 土壤 中铵态氮、硝态氮和无机氮累积量不断增加，一方 面是由于无机氮的输入，另一方面无机氮添加促进

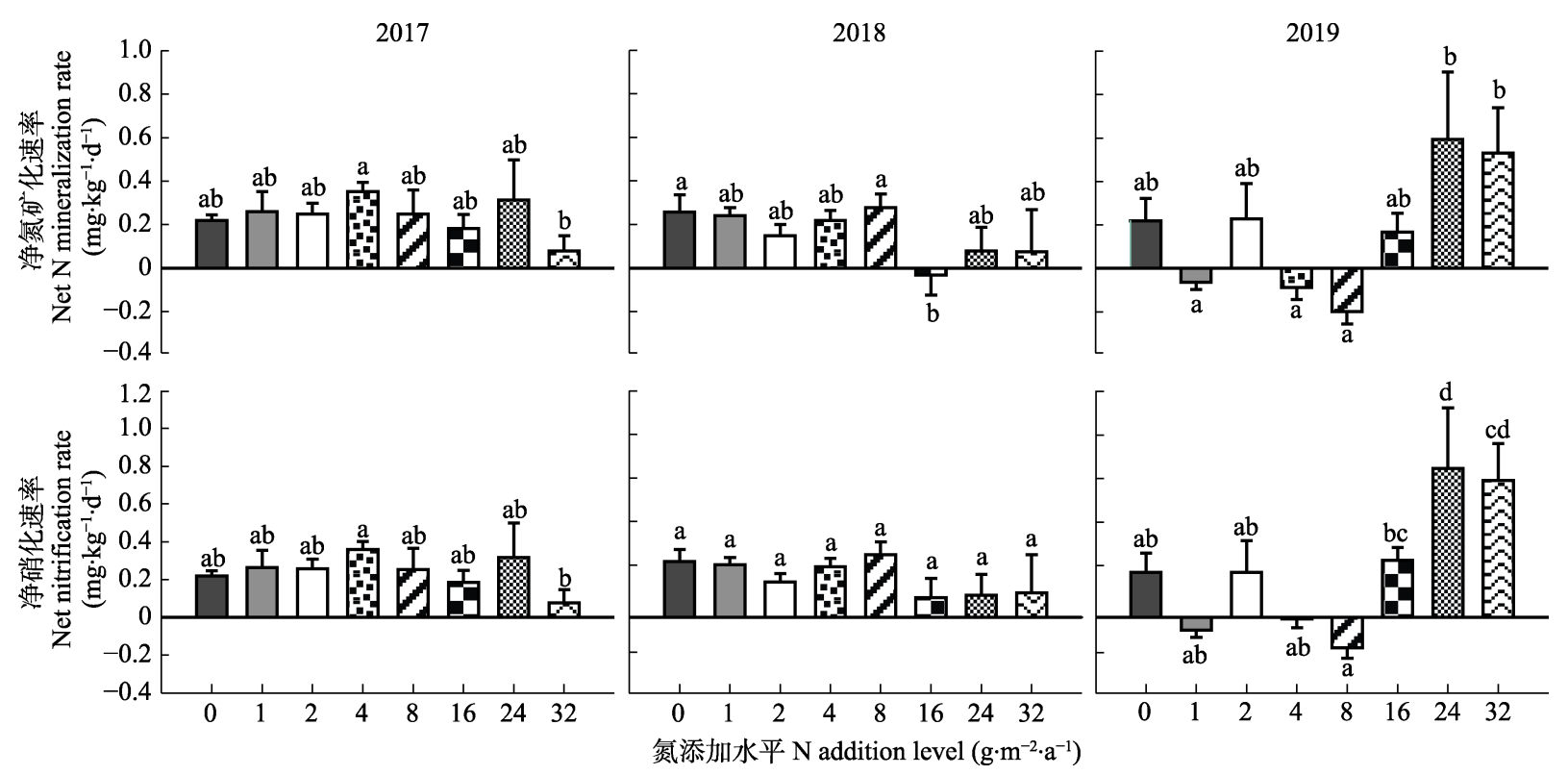

图5 华北盐渍化草地不同氮添加水平土壤净氮矿化速率和净硝化速率(平均值+标准误)。不同小写字母表示差异显著( $p<$ 0.05)。

Fig. 5 Changes in soil net nitrogen $(\mathrm{N})$ mineralization and net nitrification rates under different levels of $\mathrm{N}$ addition in a saline-alkaline grassland of northern China (mean $+S E)$. Different lowercase letters indicate significant differences $(p<0.05)$.
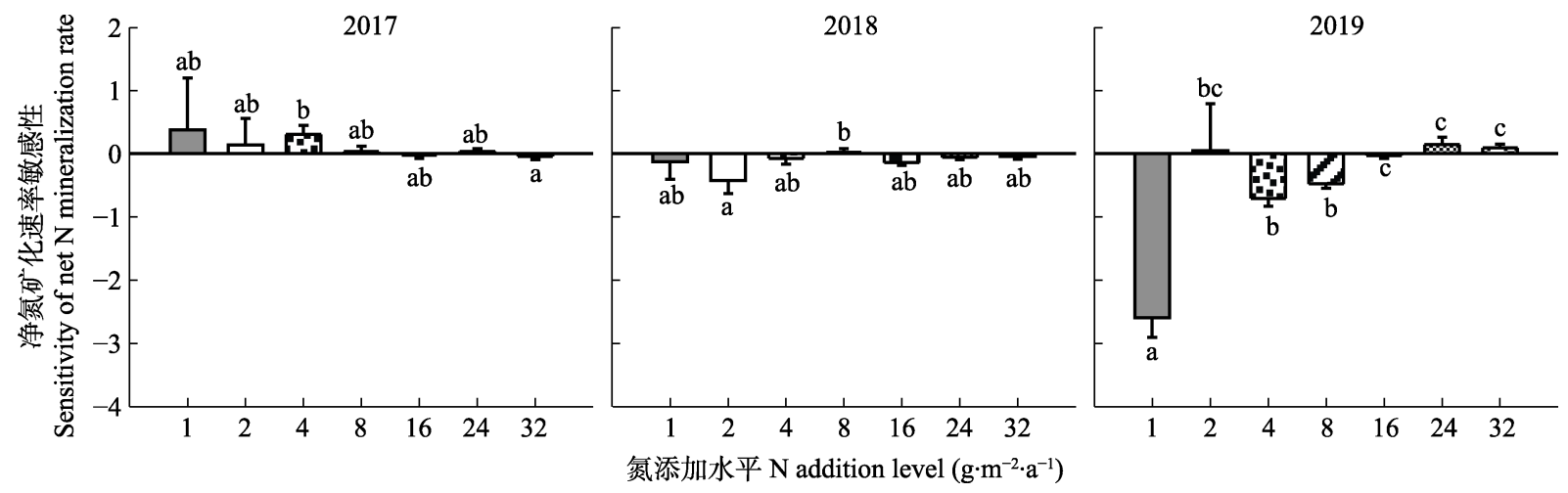

图6 华北盐渍化草地不同氮添加水平土壤净氮矿化速率的敏感性(平均值+标准误)。不同小写字母表示差异显著 $(p<0.05)$, 显著性均是以绝对值计算标注。

Fig. 6 Sensitivity of soil net nitrogen $(\mathrm{N})$ mineralization rate under different levels of $\mathrm{N}$ addition in a saline-alkaline grassland of northern China (mean $+S E)$. Different lowercase letters indicate significant differences $(p<0.05)$ in the absolute value among different treatments. 


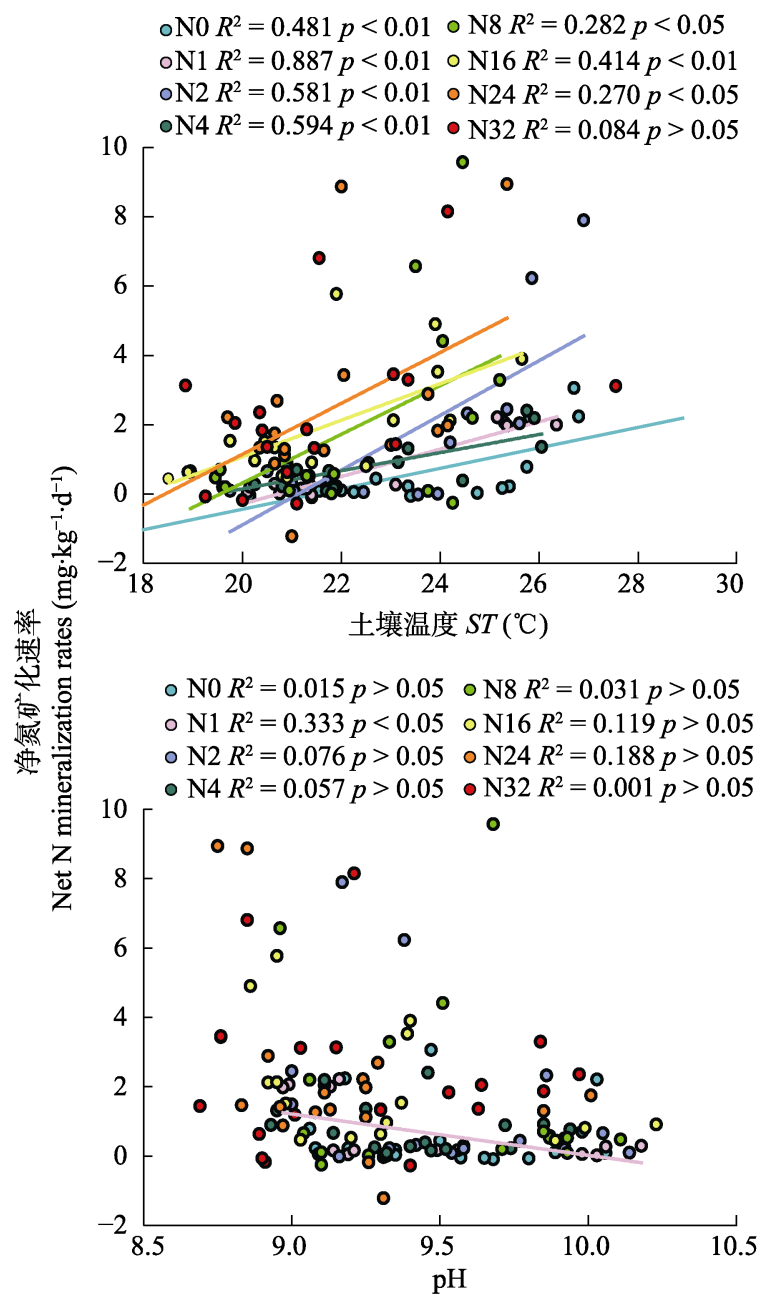

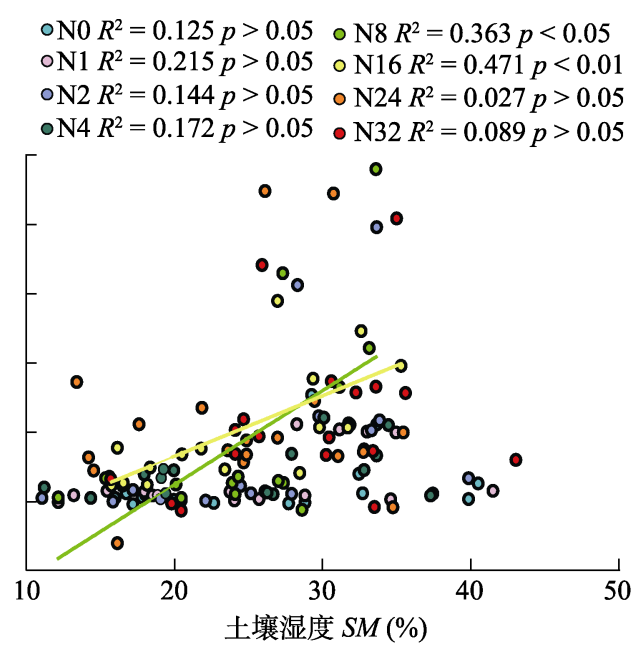

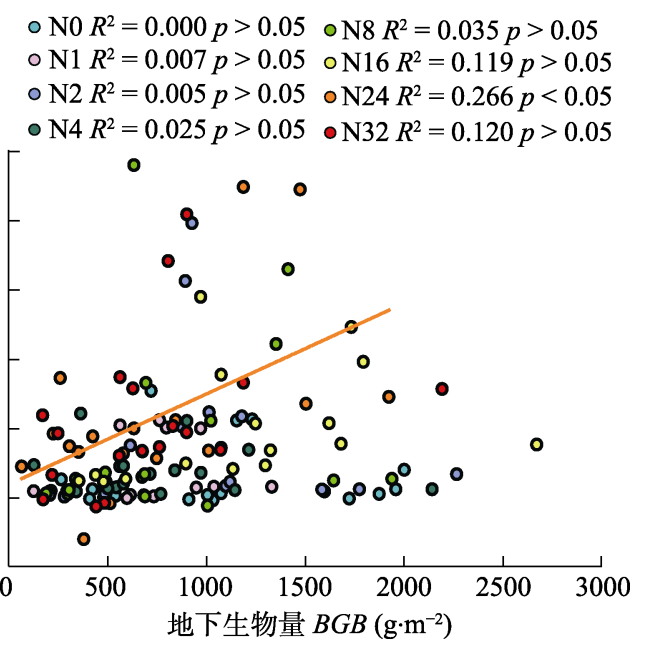

图7 华北盐渍化草地不同水平氮添加净氮矿化速率与土壤温度、湿度、土壤 $\mathrm{pH}$ 及地下生物量的相关性。 $p>0.05$ 表示净 氮矿化速率与土壤指标呈显著相关关系, $p<0.05$ 表示净氮矿化速率与土壤指标无显著相关关系。N0、N1、N2、N4、N8、 N16、N24、N32分别表示氮添加水平分别为 $0 、 1 、 2 、 4 、 8 、 16 、 24 、 32 \mathrm{~g} \cdot \mathrm{m}^{-2}$ 。

Fig. 7 Correlation between net nitrogen $(\mathrm{N})$ mineralization rates and soil temperature (ST), soil moisture (SM), $\mathrm{pH}$ and belowground biomass $(B G B)$ under different levels of $\mathrm{N}$ addition in a saline-alkaline grassland of northern China. $p>0.05$ indicated that there was a significant correlation between the net $\mathrm{N}$ mineralization rate and soil indicators; $p<0.05$ indicated that there was no significant correlation between the net nitrogen mineralization rate and soil indicators. N0, N1, N2, N4, N8, N16, N24 and N32 indicated that the nitrogen addition levels were $0,1,2,4,8,16,24$ and $32 \mathrm{~g} \cdot \mathrm{m}^{-2}$, respectively.

了土壤的净矿化和净硝化作用(刘碧荣等, 2015; Wang et al., 2015)。已有研究发现, 不同水平氮添加 对土壤总氮库没有显著响应(Liu et al., 2013; 宋冰, 2016), 但是土壤净氮矿化速率随着施氮浓度的增 加而显著上升(文汲等, 2015; 罗亲普等, 2016); 另 有研究发现土壤净氮矿化对不同施氮水平存在响应 阈值(张璐等, 2009; Chen et al., 2019; 李阳等, 2019), 即土壤净氮矿化速率没有随着施氮浓度的增加而上 升，可能的原因：长期施氮降低土壤 $\mathrm{pH}$, 高氮添加 使 $\mathrm{pH}$ 降低得更快, $\mathrm{pH}$ 降低减少了土壤有机质的可溶 性, 胞外酶代谢速率和分解速率降低(文汲等, 2015); 随着土壤中矿质氮含量的增加会影响土壤微生物腐
殖质分解酶的产生, 影响了土壤微生物活性, 因此 土壤净矿化速率降低。也有研究利用观察植物中标 记氮的回收率来进一步验证高氮添加抑制土壤净氮 矿化速率(潘庆民等, 2005)。我们的研究结果表明, 随着施氮浓度的增加土壤净矿化速率增加, 但是没 有发现阈值, 可能的原因, 一是增加的氮降低土壤 C:N (王建林等, 2014), 为土壤微生物提供了更加合 适的土壤基质(Bai et al., 2011), 适宜的温度和水分 会提高微生物种群数量和活动(Rustad et al., 2001; Wang et al., 2006), 促进了土壤中氮的释放; 二是该 研究区域属于盐渍化草地, 受养分的限制, 即使添 加氮, 养分也比较容易流失, 所以高浓度氮添加促 
进了土壤净氮矿化速率和植物对土壤有效氮的吸收, 从土壤氮的角度阐明了该研究区域生产力受氮限 制。土壤 $\mathrm{pH}$ 影响土壤净氮矿化速率, 有研究表明高 $\mathrm{pH}$ 会提高土壤基质的有效性, 净氮矿化速率会随着 $\mathrm{pH}$ 的增加而增加(Fu et al., 1987)。同时有研究表明, 土壤氮矿化速率的变化取决于土壤小气候和物种覆 盖的小尺度变化, 这些变化可以影响微生物活动和 调节土壤氮矿化(Steltzer \& Bowman, 1998; Eviner et al., 2006)。

不同水平氮添加处理下, 净氮矿化速率存在年 际差异且季节动态不同。2019年净氮矿化速率显著 高于2017和2018年, 可能是由于氮添加的累积效 应。温度和降水的差异导致不同月份之间氮的转化 速率对氮添加的响应不同, 2019年7月高氮添加 (N16、N24、N32)处理下的土壤净氮矿化速率显著 高于低氮添加处理, 降水的激发效应使得土壤微生 物生长繁殖加快, 低氮添加微生物用外源氮满足自 身需求, 所以表现为氮固持。高氮添加后, 微生物由 于自身获得足够无机氮, 代谢增强, 释放到土壤中 的无机氮含量也增加。

\section{2 土壤净氮矿化速率对不同水平氮添加的敏感性}

我们的研究结果表明, 低水平氮添加的敏感性 小于高水平氮添加的敏感性, 2019年的净氮矿化速 率较2017和2018年对低水平氮添加的敏感性更高, 可能是由于随着施氮时间的延长, 长期高水平氮添 加使得养分过于充足导致微生物逐渐适应高水平氮 添加的环境, 从而耐受性增加, 敏感性减小; 而长 期低氮添加对微生物的影响更大, 低水平氮添加后, 土壤环境达到微生物生长最适宜环境, 微生物活性 更高, 所以低氮添加时净氮矿化敏感性更高。此外, 土壤净氮矿化速率对氮添加的敏感性在年际之间也 存在显著差异, 2018年对低氮添加敏感性低是由于 微生物在该研究地缺养分缺水, 处于水分胁迫状态, 而2018年降水量高, 解除了干旱限制, 所以微生物 对低氮添加的抗性恢复, 对低氮添加的敏感性降低, 因此2018年净氮矿化对低氮添加的敏感性较2017和 2019年低。所以施肥量过低不利于提高土壤的净氮 矿化速率, 而施肥量过高, 会使土壤的敏感性减小 导致耐受性增加。由此, 盐渍化草地短期中等程度 的氮添加会提高土壤氮的可利用性和生产力。盐渍 化草地土壤无机氮含量较低, 对氮添加的缓冲性比 较弱, 外源氮输入对盐渍化草地土壤净氮矿化速率
的敏感性需要进一步深入研究。

\section{4 结论}

本研究通过分析不同水平氮添加对华北盐渍化 草地土壤净氮矿化速率的影响和净氮矿化速率对不 同水平氮添加的敏感性, 得出以下结论: 3 年短期氮 添加提高了土壤的无机氮库, 说明在养分贫㾑的盐 渍化草地外源氮的输入改变了土壤可利用养分的平 衡; 与低氮添加相比, 高水平氮添加通过提高土壤 微生物活性(N24、N32) 促进了土壤净硝化速率; 2-32 $\mathrm{g} \cdot \mathrm{m}^{-2}$ 的范围没有出现净氮矿化速率对氮添加 的响应阈值, 需要进一步从养分不平衡的角度解释 没有出现阈值的原因; 土壤净氮矿化速率对不同水 平氮添加响应的敏感性不同, 低水平氮添加的敏感 性高于高水平氮添加的敏感性, 短期中等程度的氮 添加会提高土壤氮的可利用性和生产力; 由于原位 培养实验土壤净氮矿化会受环境生物和非生物因子 的共同影响, 因此盐渍化草地土壤净氮矿化对氮添 加的响应机理和响应阈值还需要长期、深入、系统 地研究。

致谢 感谢山西右玉黄土高原草地生态系统定位观 测研究站给予本研究野外工作的支持, 感谢中国科 学院植物研究所大学生科创项目的资助。

\section{参考文献}

Aber JD, Magill AH (2004). Chronic nitrogen additions at the Harvard Forest (USA): the first 15 years of a nitrogen saturation experiment. Forest Ecology and Management, 196, 1-5.

Bai JB, Xu XL, Fu G, Song MH, He YT, Jiang J (2011). Effects of temperature and nitrogen input on nitrogen mineralization in alpine soils on Tibetan Plateau. Agricultural Science \& Technology, 12, 1909-1912.

Bao SD (2000). Analysis of Soil Agrochemical. 3rd ed. China Agriculture Press, Beijing. [鲍士旦 (2000). 土壤农化分 析. 3版. 中国农业出版社, 北京.]

Chen D, Xing W, Lan Z, Saleem M, Wu Y, Hu S, Bai Y (2019). Direct and indirect effects of nitrogen enrichment on soil organisms and carbon and nitrogen mineralization in a semi-arid grassland. Functional Ecology, 33, 175-187.

Eviner VT, Chapin III FS, Vaughn CE (2006). Seasonal variations in plant species effects on soil $\mathrm{N}$ and $\mathrm{P}$ dynamics. Ecology, 87, 974-986.

Fu MH, Xu XC, Tabatabai MA (1987). Effect of pH on nitrogen mineralization in crop-residue-treated soils. Biology and Fertility of Soils, 5, 115-119.

DOI: 10.17521/cjpe.2020.0153 
Galloway JN, Townsend AR, Erisman JW, Bekunda M, Cai ZC, Freney JR, Martinelli LA, Seitzinger SP, Sutton MA (2008). Transformation of the nitrogen cycle: recent trends, questions, and potential solutions. Science, 320, 889-892.

Guo CY, Kang JS, Wang HS (2013). Suitable Plants in Coastal Saline Alkali Land. China Construction Industry Press, Beijing. [郭成源, 康俊水, 王海生 (2013). 滨海盐碱地 适生植物. 中国建筑工业出版社, 北京.]

Hsu JS, Powell J, Adler PB (2012). Sensitivity of mean annual primary production to precipitation. Global Change Biology, 18, 2246-2255.

Jia YL, Yu GR, He NP, Zhan XY, Fang HJ, Sheng WP, Zuo Y, Zhang DY, Wang QF (2014). Spatial and decadal variations in inorganic nitrogen wet deposition in China induced by human activity. Scientific Reports, 4, 3763. DOI: 10.1038/srep03763.

Li Y, Xu XH, Sun W, Shen Y, Ren TT, Huang JH, Wang CH (2019). Effects of different forms and levels of $\mathrm{N}$ additions on soil potential net $\mathrm{N}$ mineralization rate in meadow steppe, Nei Mongol, China. Chinese Journal of Plant Ecology, 43, 174-184. [李阳, 徐小惠, 孙伟, 申颜, 任婷婷, 黄建辉, 王常慧 (2019). 不同形态和水平的氮添加对内蒙古草 甸草原土壤净氮矿化潜力的影响. 植物生态学报, 43, 174-184.]

Liu BR, Wang CH, Zhang LH, Dong KH (2015). Effect of nitrogen addition and mowing on soil nitrogen mineralization in abandoned grasslands in Inner Mongolia. Acta Ecologica Sinica, 35, 6335-6443. [刘碧荣, 王常慧, 张丽 华, 董宽虎 (2015). 氮素添加和刈割对内蒙古弃耕草地 土壤氮矿化的影响. 生态学报, 35, 6335-6343.]

Liu XR, Ren JQ, Li SG, Zhang QW (2015). Effects of simulated nitrogen deposition on soil net nitrogen mineralization in the meadow steppe of Inner Mongolia, China. PLOS ONE, 10, e0134039. DOI: 10.1371/journal.pone.0134039.

Liu YW, Xu-Ri, Xu XL, Wei D, Wang YH, Wang YS (2013). Plant and soil responses of an alpine steppe on the Tibetan Plateau to multi-level nitrogen addition. Plant and Soil, 373, 515-529.

Luo QP, Gong JR, Xu S, Baoyin T, Wang YH, Zhai ZW, Pan Y, Liu M, Yang LL (2016). Effects of N and P additions on net nitrogen mineralization in temperate typical grasslands in Nei Mongol, China. Chinese Journal of Plant Ecology, 40, 480-492. [罗亲普, 龚吉芯, 徐沙, 宝音陶 格涛, 王忆慧, 翟占伟, 潘琰, 刘敏, 杨丽丽 (2016). 氮 磷添加对内蒙古温带典型草原净氮矿化的影响. 植物 生态学报, 40, 480-492.]

Neff JC, Holland EA, Dentener FJ, McDowell WH, Russell KM (2002). The origin, composition and rates of organic nitrogen deposition: a missing piece of the nitrogen cycle? Biogeochemistry, 57-58, 99-136.

Pan QM, Bai YF, Han XG, Yang JC (2005). Effects of nitrogen additions on a Leymus chinensis population in typical steppe of Inner Mongolia. Acta Phytoecologica Sinica, 29, 311-317. [潘庆民，白永飞，韩兴国，杨景成 (2005). 氮 素对内蒙古典型草原羊草种群的影响. 植物生态学报, 29, 311-317.]

Rustad LE, Campbell JL, Marion GM, Norby RJ, Mitchell MJ, Hatley AE, Cornelissen JHC, Gurevitch J, GCTE-NEWS (2001). A meta-analysis of the response of soil respiration, net nitrogen mineralization, and aboveground plant growth to experimental ecosystem warming. Oecologia, 126, 543562.

Song B (2016). Responses of Alpine Meadow to Nitrogen Addition Gradients Test and Development of Nitrogen Saturation Theory. $\mathrm{PhD}$ dissertation, University of Chinese Academy of Sciences, Beijing. 66-67. [宋冰 (2016). 高寒 草甸生态系统对外源氮输入梯度的响应——“氮饱和” 理论的验证与发展. 博士学位论文, 中国科学院地理科 学与资源研究所, 北京. 66-67.]

Steltzer H, Bowman WD (1998). Differential influence of plant species on soil nitrogen transformations within moist meadow alpine tundra. Ecosystems, 1, 464-474.

Sun JP (2018). Study of Short-term Nitrogen Addition on Greenhouse Gases Emmisions and Its Impact Factors in Semiarid Grassland. Master degree dissertation, Shanxi Agricultural University, Taigu, Shanxi. 10. [ 孙建平 (2018). 短期氮添加对半干旱草地温室气体通量及其影 响因子的研究. 硕士学位论文, 山西农业大学, 山西太 谷. 10.]

Wang CH (2005). Soil Net $N$ Mineralization in the Typical Temperate Grassland in Inner Mongolia. PhD dissertation, Institute of Botany, Chinese Academy of Sciences, Beijing. 25-31. [王常慧 (2005). 内蒙古温带典型草原土壤 净氮矿化作用. 博士学位论文, 中国科学院植物研究 所, 北京. 25-31.]

Wang C, Butterbach-Bahl K, He N, Wang Q, Xing X, Han X (2015). Nitrogen addition and mowing affect microbial nitrogen transformations in a $\mathrm{C}_{4}$ grassland in northern China. European Journal of Soil Science, 66, 485-495.

Wang CH, Wan SQ, Xing XR, Zhang L, Han XG (2006). Temperature and soil moisture interactively affected soil net $\mathrm{N}$ mineralization in temperate grassland in Northern China. Soil Biology \& Biochemistry, 38, 1101-1110.

Wang JL, Zhong ZM, Wang ZH, Chen BX, Yu CQ, Hu XX, Shen ZX, Dacizhuoga, Zhang XZ (2014). Soil C/N distribution characteristics of alpine steppe ecosystem in Qinghai-Tibetan Plateau. Acta Ecologica Sinica, 34, 66786691. [王建林, 钟志明, 王忠红, 陈宝雄, 余成群, 胡兴 祥, 沈振西, 大次卓嘎, 张宪洲 (2014). 青藏高原高寒 草原生态系统土壤碳氮比的分布特征. 生态学报, 34, 6678-6691.]

Wen J, Yan WD, Liu YJ, Liang XC, Gao C (2015). Effect of nitrogen application on soil nitrogen mineralization of Cinnamomum camphora plantation in subtropical area.

www.plant-ecology.com 
Journal of Central South University of Forestry \& Technology, 35(5), 103-108. [文汲, 间文德, 刘益君, 梁小翠, 高超 (2015). 施氮对亚热带樟树人工林土壤氮矿化的 影响. 中南林业科技大学学报, 35(5), 103-108.]

Yu ZY, Zeng DH, Ai GY, Jiang FQ (2007). Effects of nitrogen addition on soil nitrogen availability in sandy grassland. Chinese Journal of Ecology, 26, 1894-1897. [于占源, 曾 德慧, 艾桂艳, 姜凤岐 (2007). 添加氮素对沙质草地土 壤氮素有效性的影响. 生态学杂志, 26, 1894-1897.]

Zhang L, Huang JH, Bai YF, Han XG (2009). Effects of nitrogen addition on net nitrogen mineralization in Leymus chinensis grassland, Inner Mongolia, China. Chinese Journal of Plant Ecology, 33, 563-569. [张璐, 黄建辉, 白永 飞, 韩兴国 (2009). 氮素添加对内蒙古羊草草原净氮矿 化的影响. 植物生态学报, 33, 563-569.]

Zhang NY, Guo R, Song P, Guo JX, Gao YZ (2013). Effects of warming and nitrogen deposition on the coupling mechanism between soil nitrogen and phosphorus in Songnen Meadow Steppe, northeastern China. Soil Biology \& Biochemistry, 65, 96-104.

Zhang X, Wang Q, Gilliam FS, Bai W, Han X, Li L (2012). Effect of nitrogen fertilization on net nitrogen mineralization in a grassland soil, northern China. Grass and Forage Science, 67, 219-230.

Zhu JX, He NP, Wang QF, Yuan GF, Wen D, Yu GR, Jia YL (2015). The composition, spatial patterns, and influencing factors of atmospheric wet nitrogen deposition in Chinese terrestrial ecosystems. Science of the Total Environment, 511, 777-785.

特邀编委: 戴君虎 编辑: 赵 航 